\title{
Rhythmic Mechanical Body Stimulation Improves Physical and Mental Wellbeing and Alters States of Consciousness
}

\author{
Thilo Hinterberger* and Nike Walter \\ Section of Applied Consciousness Sciences, Dept. of Psychosomatic Medicine, University Hospital of Regensburg, Regensburg, Germany
}

*Corresponding author: Thilo Hinterberger, Section of Applied Consciousness Sciences, Dept. of Psychosomatic Medicine, University Hospital of Regensburg,Franz-Josef-Strauß-Allee 11, 93053 Regensburg, Germany.

To Cite This Article: Thilo Hinterberger, Nike Walter. Rhythmic Mechanical Body Stimulation Improves Physical and Mental Wellbeing and Alters States of Consciousness. 2020 - 9(2). AJBSR.MS.ID.001368. DOI: 10.34297/AJBSR.2020.09.001368.

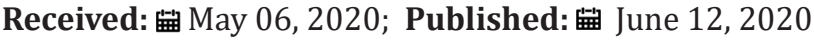

\begin{abstract}
Objectives: There is quite a debate about frequencies around $8 \mathrm{~Hz}$ playing a role in the human brain but also in micro-vibrations in muscles of the body. There are also claims about a positive bodily and mental influence of various kinds of vibrating whole-body stimulations. We have studied the effects of such a rhythmic whole-body stimulation on a subjective level.
\end{abstract}

Study Design: We tested the effects of 20 minutes mechanical whole-body stimulation at about $8 \mathrm{~Hz}$ on physical and mental wellbeing on 20 healthy participants. An additional control group of 20 participants kept the same body postures in a relaxation session.

Outcome Measures: Changes in bodily sensations, emotions and mental experiences as well as the phenomenology of consciousness were assessed by questionnaires.

Results: Compared to a control group, vibrational stimulation resulted in a significant more intense and wider body feeling and changes of the body image summarized in a factor called Integration $(\mathrm{z}=1.94, \mathrm{p}<.05)$. It also provided slightly more experiences of bliss $(\mathrm{z}=2.1$, $\mathrm{p}<.05)$. Effect sizes were moderate.

Conclusions: Despite rhythmic whole-body stimulation has an overall positive effect on body, mind and wellbeing, when compared to a relaxation exercise, it only offered a slightly more extended body awareness and stronger experiences of bliss.

Keywords: Whole body vibration training, mental wellbeing, altered states of consciousness, body rhythms

Abbreviations: PCI: Phenomenology of Consciousness Inventory; PCI-K-D: German short version of the PCI; CSP-14: Inventory assessing Changes in Subjective Self-Perception; FDR: False Discovery Rate adjustment

\section{Introduction}

There is evidence that physical stimulation through technical aids such as massage devices can have positive effects on muscular relaxation. Furthermore, such devices are also reported to have a positive influence on bodily and mental wellbeing in general. Here, we have tested this hypothesis on a rhythmic stimulation device oscillating at a speed of $7.8 \mathrm{~Hz}$ and thereby applying this vibration mechanically to the body. This frequency is termed Schumann frequency and represents a measurable electromagnetic resonance of the geosphere. This frequency is also within the brains' upper theta band (close to the alpha band from 8 to $12 \mathrm{~Hz}$ ) which shows oscillations for example in states of drowsiness, relaxation and sometimes during meditation [1]. Additionally, the shivering produced by the human muscular system is in a frequency range 4-8 Hz or even higher [2]. This tremor is also supposed to play a role in the temperature regulation of endotherm animals. There is a huge debate about the influence of artificial stimulation with this rhythm on the brain and thereby on human consciousness but also on other body functions such as the heart rate [3,4]. Despite there is some evidence for synchronization effects, this connection could 
still not be clearly scientifically answered [5]. Therefore, we used a commercial swinging plate and studied its effects on mental and physical wellbeing as well as the phenomenology of consciousness.

\section{Methods}

\section{Participants}

Each group consists of 20 participants ( 14 female and 6 male). The average age of participants in the swinging plate group was $52.2 \pm 12.5$ years and in the relaxation control group $39.6 \pm 14.3$ years. All participants gave their informed consent according to the ethical guidelines of our institutional ethics committee. None of them reported any previous illnesses or psychological disorders.

\section{Swinging Plate}

The swinging plate (Schumann $3 \mathrm{D}$ system $\AA$ ) is about $78 \mathrm{~cm}$ in size and rotates horizontally at a speed of about $7.8 \mathrm{~Hz}$ with an amplitude of less than one centimeter. Participants can either lay down on the plate or sit on a firm cushion and feel the vibrations through their back or the feet upwards.

\section{Procedure}

Participants were asked to first sit on the vibrating plate for a duration of $5 \mathrm{~min}$. followed by $5 \mathrm{~min}$. of unstimulated resting. Then, the participants lay down with the back on the plate with their legs elevated and placed on a cushion at an approximate angle of $90^{\circ}$ upright. Again, the plate was turned on for $5 \mathrm{~min}$. followed by a 5 min. resting phase. To exclude the effect of the mere resting procedure in these body positions, the procedure was repeated with a control group which was asked to sit and lay down on the floor for the same amount of time without the swinging plate. At the end, all participants filled in response questionnaires. The study has been conducted in accordance with the Declaration of Helsinki as revised in 2013.

\section{Questionnaires}

PCI-K-D: The Phenomenology of Consciousness Inventory (PCI) is a self-report measure to quantify states of consciousness after specific interventions [6]. We used a modified short German version (PCI-K-D) with 27 items out of the original 53 items, maintaining the topics of its 12 major dimensions. A factorial analysis of this short form on a sample of 159 participants from studies with various types of interventions could merge 14 items into three major dimensions. The remaining 13 items were treated as separate dimensions. The three major dimensions were termed 'Bliss' (9 items, Cronbachs alpha (Ca)=.88), 'Expansion' (2 items, $\mathrm{Ca}=.80$ ), and 'Cognitive Control' (3 items, $\mathrm{Ca}=.71$ ).

CSP-14: The CSP-14 allows for the assessment of pre-post changes in body sensation, emotional state and mental state after interventions and consists of three factors, namely (1) Integration, (2) Balance and (3) Vitality [7]. The questionnaire contains 14 items, which are rated on a scale ranging from -3 to +3. Additionally, the participants where asked for disturbance during the session, and evaluated the contact to the instructor and the duration of the intervention.

\section{Statistical Analysis}

Non-parametrical tests were used for statistical analysis. For the comparison of the two groups the Mann-Whitney U-test was calculated. To test the single-groups against zero (i.e. no change after the session in the CSP-14) a Wilcoxon signed rank test was applied. All p-values were corrected for multiple testing using False Discovery Rate (FDR) adjustment. The three CSP-14 dimensions were corrected separately and also the 17 subsequent items. The PCI-K-D was corrected with all 16 item factors.

\section{Results}

\section{Group comparisons}

Focusing on the mean values of pre-post changes, all three CSP14 dimensions as well as most of the CSP-14 items yielded highly significant changes even after FDR adjustment in both groups, the relaxation exercise and the swinging plate session (Table 1). Thus, both interventions can be characterized as a widening, intensifying, relaxing, comfortable, powerful, calming, balancing, uplifting, satisfying, secure, connecting, fulfilling, clearing, and vitalizing experience. However, the differences between swinging plate and relaxation exercise only showed an uncorrected significant difference in the main dimension Integration $(\mathrm{z}=1.94, \mathrm{p}=.026)$ but not in Balance and Vitality. This effect can be attributed to the swinging plate offering a significantly wider and more intense body perception and a more awake and clear mental state. Those differences reached moderate effect sizes between .3 and .5 .

Table 1: CSP-14 with three major dimensions and specific items. The first four columns report mean ratings and last three columns the results of a Mann-Whitney U-test between swinging plate and relaxation exercise.

\begin{tabular}{|c|c|c|c|c|c|c|c|}
\hline \multirow{2}{*}{$\begin{array}{c}\text { CSP-14 } \\
\text { Dimension: Question }\end{array}$} & \multicolumn{2}{|c|}{ Swinging Plate } & \multicolumn{2}{|c|}{ Relaxation Exercise } & \multicolumn{3}{|c|}{ Difference (U-Test) } \\
\hline & Mean & SD & Mean & SD & $\mathrm{z}$ & $\mathrm{p}$ & Effect-size $r$ \\
\hline Integration (I) & $1,76^{* *}$ & 0,92 & $1,22^{* *}$ & 0,66 & 1.94 & 0.026 & 0.307 \\
\hline Balance (B) & $1,53 * *$ & 1,36 & $1,68^{* *}$ & 0,67 & 0.26 & 0.397 & 0.041 \\
\hline Vitality (V) & $0,91^{* *}$ & 1,14 & $0,77^{* *}$ & 0,76 & -0.01 & 0.495 & -0.002 \\
\hline I: narrow- wide & $1,85^{* *}$ & 1,14 & $1,20 * *$ & 1,01 & 2.04 & 0.021 & 0.323 \\
\hline
\end{tabular}




\begin{tabular}{|c|c|c|c|c|c|c|c|}
\hline I: weaker- more intensive & $2,10^{* *}$ & 0,85 & $1,05^{* *}$ & 1,05 & $3.05^{*}$ & 0.001 & 0.483 \\
\hline I: tense- relaxed & $2,15^{* *}$ & 0,99 & $2,15^{* *}$ & 0,67 & 0.44 & 0.33 & 0.069 \\
\hline I: uncomfortable-comfortable & $1,85^{* *}$ & 1,14 & $1,50 * *$ & 1,10 & 1.05 & 0.147 & 0.166 \\
\hline V: powerless- powerful & $1,15^{* *}$ & 1,18 & $0,90^{* *}$ & 1,21 & 0.56 & 0.288 & 0.088 \\
\hline B: activated- calm & $1,55^{* *}$ & 1,57 & $1,90^{* *}$ & 0,64 & 0 & 0.5 & 0 \\
\hline B: unbalanced- balanced & $1,50^{* *}$ & 1,24 & $1,45^{* *}$ & 0,83 & 0.32 & 0.373 & 0.051 \\
\hline V: sad- happy & $1,05^{* *}$ & 1,23 & $0,75^{* *}$ & 1,02 & 0.75 & 0.226 & 0.119 \\
\hline V: unsatisfied- satisfied & $1,15^{* *}$ & 1,31 & $1,35^{* *}$ & 1,04 & -0.82 & 0.207 & -0.129 \\
\hline I: anchorless- secure & $1,15^{* *}$ & 1,31 & $0,75^{* *}$ & 0,79 & 0.68 & 0.248 & 0.107 \\
\hline I: dissociated-connected & $1,50^{* *}$ & 1,24 & $1,05^{* *}$ & 1,05 & 1.17 & 0.122 & 0.184 \\
\hline I: tired, confused--awake, clear & $1,70^{* *}$ & 1,26 & $0,85^{*}$ & 1,46 & 1.88 & 0.03 & 0.297 \\
\hline $\mathrm{V}$ : introverted- extroverted & 0,30 & 1,53 & 0,15 & 1,31 & 0.25 & 0.401 & 0.04 \\
\hline V: empty-fulfilled & $0,90 *$ & 1,62 & $0,70^{* *}$ & 0,92 & 0.27 & 0.394 & 0.043 \\
\hline something disturbing & 0,25 & 0,44 & 0,00 & 0,00 & 2.34 & 0.01 & 0.369 \\
\hline bad contact - good contact & $2,60^{* *}$ & 0,82 & $2,95^{* *}$ & 0,22 & -1.77 & 0.039 & -0.279 \\
\hline too short- too long & $-0,65$ & 1,50 & 0,00 & 0,79 & -1.71 & 0.043 & -0.271 \\
\hline
\end{tabular}

Bold: $p<.05$ one-tailed Mann-Whitney U-test, means were tested with t-test for H0: mean >0. FDR-adjusted $p$-values ${ }^{*} p<.05,{ }^{* *} p<.01$.

According to Table 2 presenting the results of the PCI-K-D the conscious experiences in both groups were blissful (F1), expanding (F2), but also characterized with a high amount of cognitive control (F3). Feeling of anger, sadness, and fear were almost not present and only a small amount of tension and sexual feelings were reported. In terms of time perception, the swinging plate offered a timeless state, whereas the relaxation exercise was also rated as decelerating. This deceleration was the only significant difference after FDR adjustment ( $\mathrm{z}=3.14$, padj<.05). Both interventions led to a high amount of imagery and remembrance. Without FDR correction the swinging plate resulted in a more blissful $(\mathrm{z}=2.10, \mathrm{p}<.05)$ and expanding $(\mathrm{z}=1.79, \mathrm{p}<.05)$ state of consciousness compared to the pure relaxation. Figure 1 illustrates the major findings by displaying the main factors of the two questionnaires.
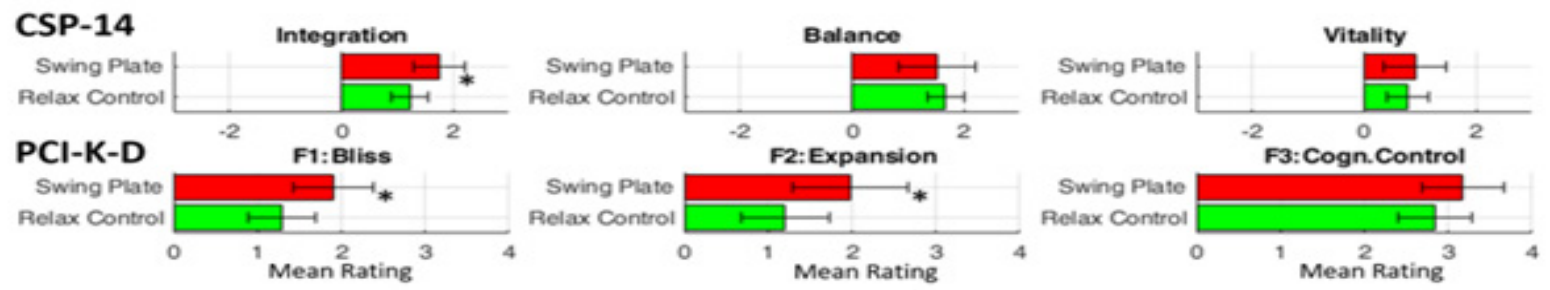

Figure 1: Mean responses in main dimensions of CSP 14 and the $\mathrm{PCl}$ questionnaire.

*significant differences between groups with $p<.05 . \mathrm{N}=20$ in each group.

Table 2: Group comparisons of the responses from the PCI-K-D.

\begin{tabular}{|c|c|c|c|c|c|c|c|}
\hline PCI-K-D & \multicolumn{2}{|c|}{ Swinging plate } & \multicolumn{2}{|c|}{ Relaxation exercise } & \multicolumn{3}{c|}{ Difference (U-Test) } \\
\hline Factor & Mean & SD & Mean & SD & z & p & Effect-size r \\
\hline F1: Bliss & $\mathbf{1 . 9 1}^{* *}$ & 0,97 & $\mathbf{1 . 2 9 * *}$ & 0,81 & $\mathbf{2 . 1}$ & 0.018 & 0,332 \\
\hline F2: Expansion & $\mathbf{1 . 9 8}^{* *}$ & 1,37 & $\mathbf{1 . 2 0 ^ { * * }}$ & 1,06 & $\mathbf{1 . 7 9}$ & 0.037 & 0,283 \\
\hline F3: Cogn. Control & $\mathbf{3 . 1 8}^{* *}$ & 0,98 & $\mathbf{2 . 8 3} * *$ & 0,88 & 1.3 & 0.096 & 0,206 \\
\hline Anger & 0 & 0,00 & 0.05 & 0,22 & -0.95 & 0.171 & $-0,150$ \\
\hline Sadness & 0.1 & 0,45 & 0.05 & 0,22 & 0 & 0.5 & 0,000 \\
\hline Fear & 0.1 & 0,45 & 0 & 0,00 & 0.95 & 0.171 & 0,150 \\
\hline Timeless & $\mathbf{2 . 0 0 * *}$ & 1,45 & $\mathbf{2 . 1 0} * *$ & 1,07 & -0.06 & 0.478 & $-0,009$ \\
\hline
\end{tabular}




\begin{tabular}{|c|c|c|c|c|c|c|c|}
\hline Acceleration & $0.80 * *$ & 1,06 & $0.40 *$ & 0,60 & 1.03 & 0.151 & 0,164 \\
\hline Deceleration & $0.75^{*}$ & 1,25 & $2.20^{* *}$ & 1,36 & $-3.14^{*}$ & 0.001 & $-0,497$ \\
\hline Imagination & $1.50^{* *}$ & 1,61 & $1.75^{* *}$ & 1,41 & -0.6 & 0.275 & $-0,095$ \\
\hline Distraction & $0.70^{*}$ & 0,98 & $0.95^{* *}$ & 1,00 & -0.75 & 0.227 & $-0,118$ \\
\hline Soliloquy & $0.80 *$ & 1,24 & $0.50 *$ & 0,83 & 0.57 & 0.284 & 0,090 \\
\hline Remembrance & $3.30 * *$ & 1,17 & $2.35^{* *}$ & 1,53 & 2.35 & 0.009 & 0,372 \\
\hline Tension & 0.45 & 0,83 & $0.40^{*}$ & 0,68 & 0.14 & 0.443 & 0,023 \\
\hline Sexual feelings & 0.3 & 0,92 & 0.15 & 0,49 & 0.45 & 0.327 & 0,071 \\
\hline Extroversion & $0.90 * *$ & 0,91 & $1.20 * *$ & 1,01 & -0.95 & 0.171 & $-0,150$ \\
\hline
\end{tabular}

Bold: $\mathrm{p}<.05$ one-tailed Mann-Whitney U-test, means were tested with t-test for H0: mean $>0$. FDR-adjusted $p$-values ${ }^{*} p<.05,{ }^{* *} p<.01$.

\section{Discussion}

The goal of this study was to evaluate subjective changes of wellbeing and describe conscious experiences which might be specific to a rhythmic mechanical whole-body stimulation. We have found that most of the positive effects on subjective wellbeing we measured were already present in a simple relaxation session offered in these lying and sitting positions. However, some experiences such as the wider and more intense body sensation were significantly more enhanced after vibrational stimulation. This effect is plausible because tactile whole-body stimulation increases the bodyoriented mindfulness leading to an enhanced mental body-image. It still remains questionable to what extent the resulting changes in body sensation could be ascribed to the stimulation frequency and also to the enhancement of the intrinsic physiologic microvibrations. Other studies reported whole body vibration training having positive effects on muscle strength, cardiovascular health, blood flow [8], as well as quality of life in patients with chronic conditions [9]. However, multiple whole-body vibration techniques exist and effects vary with different frequencies of vibrations [10]. Thus, the specific characteristic of the vibration at $7.8 \mathrm{~Hz}$ needs further investigation in order to illuminate its potential application in health. There is actually one hint towards the $7.8 \mathrm{~Hz}$ stimulation positively influencing the brain's theta rhythms which could explain the increased perception of states of bliss, love and joy during stimulation. However, this hypothesized connection still has to be investigated by simultaneous EEG measurements in future.

Importantly, the aim of this study was not to evaluate the commercial claims made by companies selling swinging plates. To this end, no statement can be made regarding the possible healthsupporting effects because the main positive effects showing a superiority to a normal relaxation session do not survive a correction for multiple testing. On the other hand, the effect sizes demonstrating the superiority of the swinging plate in terms of offering a more intense, widening, and blissful experience are already moderate in size.

\section{Limitations}

The primary limitation of this particular study is the small sample size as well as the difference in the significant mean age of the groups $(\mathrm{z}=2.48, \mathrm{p}<.01)$, nevertheless yielding moderate effect sizes in the group comparison. Further, the questionnaire PCI-K should be validated in further studies.

\section{Conclusion}

To conclude, the results of this study present the positive effects of a rhythmic whole-body stimulation on changes in body sensation, emotional and mental state as well as the phenomenology of consciousness. However, the impact of the Schumann frequency as stimulation frequency still needs further investigations.

\section{Acknowledgements}

The authors thank Club of Wellness (CoW) Health Technologies (Germany) for providing the Schumann 3D system. The authors received no specific funding for this work.

\section{References}

1. Cahn BR, Polich J (2006) Meditation states and traits: EEG, ERP, and neuroimaging studies. Psychological Bulletin 132(2): 180-211.

2. Blondin DP, Haman F (2018) Shivering and nonshivering thermogenesis in skeletal muscles. Handbook of Clinical Neurology 156: 153-173.

3. Saroka KS, Vares DE, Persinger MA (2016) Similar Spectral Power Densities Within the Schumann Resonance and a Large Population of Quantitative Electroencephalographic Profiles: Supportive Evidence for Koenig and Pobachenko. PloS One 11(1): e0146595.

4. McCraty R, Atkinson M, Stolc V, Alabdulgader AA, Vainoras A, et al. (2017) Synchronization of Human Autonomic Nervous System Rhythms with Geomagnetic Activity in Human Subjects. Int J Environ Res Public Health 14(7): 770.

5. Usui T, Sakamoto K, Okuno H (1984) Mechanism of mechanical vibrations in living body and its application to various fields. The Annals of physiological anthropology 3(3): 177-193.

6. Pekala R (1991) Quantifying Consciousness: An Empirical Approach. New York, NY: Plenum Press.

7. Hinterberger T, Baierlein F, Breitenbach N (2018) Skin Conductance Feedback meditation: Evaluation of a novel physiology-assisted meditation style. Complementary Medicine Research 25: 313-320. 
8. Games KE, Sefton JM, Wilson AE (2015) Whole-body vibration and blood flow and muscle oxygenation: a meta-analysis. Journal of Athletic Training 50(5): 542-549.

9. Li G, Zhang G, Wang Y, Wang X, Zhou H, et al. (2019) The effect of wholebody vibration on health-related quality of life in patients with chronic conditions: a systematic review. Quality of Life Research 28(11): 2859. 2870 .
10. Cardinale M, Wakeling J (2005) Whole body vibration exercise: are vibrations good for you? Br J Sports Med 39(9):585-589. 